\title{
Cluster geometry and inclinations from deprojection uncertainties
}

\section{Cluster geometry and inclination}

\author{
D. Chakrabarty ${ }^{1}$, E. De Filippis ${ }^{2}$, and H. Russell ${ }^{3}$ \\ 1 School of Physics \& Astronomy, University of Nottingham, Nottingham NG7 2RD, UK \\ e-mail: dalia.chakrabarty@nottingham.ac.uk \\ 2 Department of Physical Sciences, University of Napoli Federico II, via Cintia 6, 80126 Napoli, Italy \\ e-mail: betty@na.infn.it \\ 3 Institute of Astronomy, University of Cambridge, Madingley Road, Cambridge CB3 OHA, UK \\ e-mail: hrr27@ast.cam.ac.uk
}

Received 4 February 2008 / Accepted 30 May 2008

\section{ABSTRACT}

\begin{abstract}
Context. The determination of cluster masses is a complex problem that would be aided by information about the cluster shape and orientation (with respect to the line-of-sight).

Aims. It is in this context, that we have developed a scheme for identifying the intrinsic morphology and inclination of a cluster, by looking for the signature of the true cluster characteristics in the inter-comparison of the different deprojected emissivity profiles (that all project to the same X-ray brightness distribution) and complimenting this with SZe data when available.

Methods. We deproject the cluster X-ray surface brightness profile under assumptions about geometry and inclination that correspond to four extreme scenarios; the deprojection is performed by the non-parametric algorithm DOPING. The formalism is tested with model clusters and is then applied to a sample of 24 clusters. While the shape determination is possible by implementing the X-ray brightness alone, the estimation of the inclination is usually markedly improved upon by the usage of SZe data that is available for the considered sample.

Results. We spot 8 prolate systems, 1 oblate and 15 of the clusters in our sample as triaxial. In fact, for systems identified as triaxial, we are able to discern how the three semi-axis lengths compare with each other. This, when compounded by the information about the line-of-sight extent, allows us to constrain the intrinsic axial ratios and the inclination quite tightly.
\end{abstract}

Key words. methods: analytical - galaxies: clusters: general

\section{Introduction}

The identification of the three dimensional cluster shape is of significant importance in the pursuit of quantities of cosmological interest. In particular, the misinterpretation of the true cluster geometry can affect the extraction of the Hubble constant from X-ray and SZe information (Zaroubi et al. 2001) and of the cluster mass. Erroneous cluster masses are often the cause of less reliable understanding of cluster physics and poorer constraints from cluster cosmology. A mandatory exercise that should be undertaken towards the extraction of the correct cluster masses is the identification of the correct geometry and inclination of the cluster. However, the lack of knowledge about these attributes are typically bypassed by resorting to the assumption of sphericity, even when the projected ellipticity of the system is indicated to be non-zero from observations (Fabian et al. 1981; Yoshikawa \& Suto 1999; Pizzolato et al. 2003). This is also the adopted geometry in frequently used packages for determining dark matter distributions in clusters, like JACO (Mahdavi et al. 2007).

On the contrary, halos realised in cosmological simulations have been found to be flattened and triaxial in shape; the distribution of ellipsoidal shapes of simulated halos have been discussed by Frenk et al. (1988); Dubinski \& Carlberg (1991); Warren et al. (1992); Cole \& Lacey (1996); Bailin \& Steinmetz (2005), among others. Moreover, the formation of large scale structure has a bearing on the intrinsic shape of cluster sized halos
(Plionis et al. 2004); thus, the 3D cluster morphology could be a device that can be used to constrain cosmological models.

While the statistical analysis of the 3D cluster morphology has been looked into (see Jing \& Suto 2002; Kasun \& Evrard 2005; Hayashi et al. 2007, and references therein), the determination of the full 3D shapes and orientations of individual halos is a harder problem that has attracted relatively less attention (Sereno et al. 2006).

Recovery of the full 3D morphology of a cluster, using measurements of accessible cluster characteristics (such as X-ray emission profiles or 2D X-ray surface brightness distributions or SZe data), would require expertise in deprojecting such observed information into the full 3D distribution, under general triaxial geometries. Such can be achieved via parametric fits; in fact, parametric deprojection of the observed optical surface brightness maps of galaxies, under assumptions of sphericity and (more rarely) axisymmetry, has been studied before (Palmer 1994; Bendinelli 1991). However, the fundamental problems with parametric fits are (i) the answer depends on the choice of the parametrisation; and (ii) the goodness-of-fit quantifier (such as a $\chi^{2}$ measure) can appear spuriously inflated, particularly in the presence of non-homogeneous measurement noise (Bissantz \& Munk 2001)

Thus, non-parametric deprojection is a better option. However, deprojection algorithms that promise improved three dimensional cluster mass distributions, by taking the measured 
ellipticity and the true cluster morphology and orientation into account, are limited in availability. Zaroubi et al. (2001) report on the application of a non-parametric deprojection algorithm that assumes axisymmetry, to a set of simulated clusters. However, as the authors state, the applicability of this scheme to the current state of data sets appears difficult.

A comprehensive approach that calls for the amalgamation of two or more mass indicators include the implementation of X-ray observations, SZe information, lensing results and dynamical measurements. Such exercises have been undertaken already (Zaroubi et al. 1998; Reblinsky 2000; Sand et al. 2002). An attempt has been made in the recent past (Sereno et al. 2006) to decipher the three dimensional morphology and inclination distribution of a sample of clusters from Reese et al. (2002), with the aim of improving the cluster mass estimation (from X-ray measurements) more accurately. This work employs the rudimentary SZe temperature decrement data (De Filippis et al. 2005) in the $\beta$-modelling of the cluster. Thus, this work is susceptible to very large error bars that currently plague SZe data. This is of course topped by the errors that can be introduced by the choice of ellipsoids of revolution that are implemented to model their sample clusters.

In this work, we present a novel, model-independent trick to determine the correct morphology and inclination of a cluster, without resorting to the assumption of axisymmetry. Such a determination is made possible by analysing the multiple deprojected emissivity or X-ray luminosity density distributions that are recovered under distinct assumptions about the cluster geometry and inclination and that will all project to the same observed X-ray brightness map. Thus, the deprojection in question needs to be performed by a scheme that is efficient in carrying out deprojection under general geometries, at any assumed inclination. This is possible with a new inverse deprojection algorithm DOPING (Deprojection of Observed Photometry using an INverse Gambit) that has been reported in Chakrabarty \& Ferraese (2008).

The inter-comparison of the amplitudes and shapes of the different deprojected emissivity distributions tell us the correct shape of the cluster (including triaxial ones) while the inclination constraints are improved upon by the implementation of the available cluster elongation information from the SZe data, as reported in the literature. In case of triaxial systems, the SZe data is used to determine the two intrinsic axial ratios.

The paper is organised as follows. Following the first introductory section, we discuss the deprojection algorithm in brief. Section 3 is devoted to the methodology that we use to extract the cluster shapes from the deprojected profiles. The testing of the advanced scheme is dealt within the following section. We then proceed to apply this method to our cluster sample (Sect. 5), which precedes the results section. The paper is rounded off by recounting some relevant aspects of the presented work.

\section{DOPING}

DOPING stands for Deprojection of Observed Photometry using an INverse Gambit. However, the name does not do full justice to the capacity of the algorithm; it is a deprojection algorithm that could just as well be applied to deproject observed X-ray 2D surface brightness maps $(S B)$ of clusters. The deprojection can be performed in general triaxial geometries and is able to incorporate radial variations in shape. The deprojected luminosity density distribution is sought by penalised likelihood approach that uses an MCMC optimiser. Although DOPING is in general able to use the 2D $S B$ information and provide the full three dimensional $\rho$, in the case of an oblate/prolate system with a uniform projected eccentricity, the effective dimensionality of the problem reduces by one, since the projected and intrinsic eccentricities can then be related through analytical relations. This idea is exploited in the work. The $S B$ is treated as a function of distance along a photometric axis (the $x$-axis). Similarly, the emissivity or X-ray luminosity density distributions are presented along the $x$-axis.

\section{Method}

As explained in Sect. 2, DOPING is able to incorporate information about the LOS extent of a cluster from SZe measurements, in the process of deprojecting under the assumption of triaxiality. However, the poor quality of the currently available SZe data will cause the determination of shape ambiguous. Thus, for the current purpose, we will be carrying out the deprojection of the observed X-ray $S B$ under assumptions of prolateness or oblateness for the intrinsic shape of the observed cluster; as for its inclination, we choose to deproject at the two ends of the range of inclinations allowed for an oblate geometry (discussed below). The other, even more important reason for choosing to deproject at these geometry+inclination configurations is the relative simplicity of deprojection under oblateness/prolateness than triaxiality.

The different X-ray luminosity density distributions or the emissivity distributions $(\rho)$ that will be recovered from such deprojections, will be analysed to tell us if the system under consideration is prolate, oblate or even triaxial.

Now, let us examine our choice of inclinations for deprojection, in greater detail. We will carry out the deprojections under the assumptions of $i=90^{\circ}$ and $i=i_{\min }$, where $i_{\min }$ is the smallest allowed inclination for the measured projected eccentricity for the assumption of oblateness $\left(i_{\min }=\sin ^{-1} e_{\mathrm{p}}\right.$, where $e_{\mathrm{p}}$ is the projected eccentricity that is related to the projected axial ratio $q_{\mathrm{p}}$ as $e_{\mathrm{p}}^{2}=1-1 / q_{\mathrm{p}}^{2}$ ). As a result of such deprojection of an $S B$, we will be able to say if the cluster is inclined to the lineof-sight (LOS) by an angle that is less than $i_{\min }$ or is of a value intermediate to $90^{\circ}$ and $i_{\min }$.

Thus, there are 4 distinct deprojection configurations $(D)$ that we will consider for any given SB:

1. assumed geometry is prolateness and assumed $i=90^{\circ}: D_{90}^{\mathrm{p}}$;

2. prolateness and $i=i_{\min }: D_{\min }^{\mathrm{p}}$;

3. oblateness and $i=90^{\circ}: D_{90}^{\mathrm{o}}$; and

4. oblateness and $i=i_{\min }: D_{\min }^{\mathrm{o}}$.

On the basis of these deprojections, we will identify the shape and inclination of a given cluster.

\section{Effect of $D$}

It is to be emphasised that our determination of the true 3D shape of a cluster is not affected by the exact geometry+inclination configuration that we choose to deproject its X-ray $S B$ under; rather, it is the intercomparison of the various profiles of $\rho$ that are recovered from deprojection under the different geometry+inclination configurations that we choose. Thus, if we choose to deproject an observed X-ray $S B$, assuming the cluster to have a geometry+inclination different from the ones that we choose, then the inter-comparison of the corresponding profiles of $\rho$ will be different from what we infer here. It is a question of standardising such an inter-comparison, in order to be able to 
conclude the true cluster characteristics. All we do in this paper is advance the same, using the trends in the inter-comparison that we notice, when the cluster at hand is deprojected under our choices of geometry+inclination, i.e. $D$. The modus-operandi that connects such an inter-comparison to the true cluster characteristics, is discussed below (Sect. 3.2).

\subsection{Coordinate system}

The two coordinate systems that suggest themselves readily to any projection related investigation are the body coordinates of the observed system (considered regular Cartesian, marked here in upper case letters $X-Y-Z$ ) and the observer's coordinate system (marked in lower case $x-y-z$ ). The POS is spanned by the $x-y$ plane while $z$ is along the LOS. Also, we consider one of the principal axes of the cluster $(X)$ to be coincident with one of the photometric axes $x$ (the photometric major axis for oblate models and the photometric minor axis for the prolate case).

\subsection{Underlying principle}

The determination of a cluster's intrinsic shape and inclination is based on the fact that the inter-comparison of $\rho$ recovered by deprojecting under the assorted geometry and inclination configurations $D$, bears the signature of the true characteristics of the cluster.

The comparison of (the shape and amplitude of) two distinct profiles of $\rho$ that will be recovered from deprojecting a given $\mathrm{X}$-ray $S B$, under two distinct deprojection scenarios (2 different $D s)$, will depend on the inter-comparison of the

- extents along the LOS, of the 2 systems that are ascribed to the 2 different assumptions of geometry (corresponding to the $2 D s$ )

- ellipsoidal radii to the same generic point $(x, y, z)$, in the 2 systems described by the $2 \mathrm{Ds}$, assuming the gas density to be stratified on concentric ellipsoidal shells.

In this section, these ideas are expounded upon.

\subsubsection{Effect of varying the LOS extent}

The luminosity density that is required to be projected through a given length, in order to produce an observed $S B$, must be higher when the extent along the axis of projection is less in a deprojection model than in the case of the "true system", i.e. the observed cluster. In other words, $\operatorname{LOS}_{\mathrm{D}}<\operatorname{LOS}_{\mathrm{T}} \Longrightarrow \rho_{\mathrm{D}}>\rho_{\mathrm{T}}$. Here, the subscript " $D$ " refers to quantities relevant to the deprojection model while the subscript "T" refers to quantities relevant to the true system. (We will follow this notation elsewhere in the paper too.)

\subsubsection{Effect of varying the 3-D density structure}

We can develop a qualitative understanding of the shapes of the recovered density profiles by assuming that the density is given as a function of the ellipsoidal radius $\xi$. The form of $\xi$ is determined by the geometry and inclination $i$ that describe a system, as well as its intrinsic eccentricity $e$. Thus, for an oblate system, inclined at angle $i$, for any $(x, y, z), \xi$ is:

$$
\begin{aligned}
& x^{2}+y^{2}\left(\cos ^{2} i+\frac{\sin ^{2} i}{1-e^{2}}\right)+z^{2}\left(\sin ^{2} i+\frac{\cos ^{2} i}{1-e^{2}}\right) \\
&-y z \sin 2 i \frac{e^{2}}{1-e^{2}}=\xi^{2} .
\end{aligned}
$$

Thus, we assume $\rho=\rho(\xi)$. We also assume that the physically motivated idea of monotonicity holds true in the density distribution: $\rho\left(\xi_{i}\right)<\rho\left(\xi_{j}\right), \forall \xi_{i}>\xi_{j}$. At this stage, this exercise is held independent of effects of LOS extent on the density.

Now, let us consider two systems with different $e, i$ and geometries.

- Let the systems be such that in System I, the ellipsoidal radius to a general point $x, y, z$ is higher than in System II, i.e. to $x, y, z, \xi_{I}>\xi_{I} I$ (say).

- Then $\rho_{I}(x, y, z)<\rho_{I} I(x, y, z)$, since we assume density to monotonically fall with $\xi$.

- Since the projection of density (observed $S B$ ) is given from measurement, to compensate for this trend, in System I, a given value of density would show up at a higher $\xi$, than in System II.

This translates to the given density showing up at a higher $x$, in System I than System II, when the density profile along the $\hat{x}$-direction is sought.

Now let us replace System II with the true system and let System I be one of the 4 deprojection models that we consider. Thus, in this case, $\xi_{\mathrm{D}}>\xi_{\mathrm{T}}$. This comparison can imply stronger effects on the density at smaller values of $x$ or larger values of $x$, depending on the relative geometries of the two systems in question. While such situations are explored below, now let us monitor the consequences of $\xi_{\mathrm{D}}$ exceeding $\xi_{\mathrm{T}}$, first near the core of the cluster and then at the outer parts of the cluster.

When $\xi_{\mathrm{D}}>\xi_{\mathrm{T}}$ around the core, it has the following effect on the shape of the recovered density profile. If the density profile of the true system is as shown in solid lines on the left in Fig. 2, then the recovered density profile will be flatter at the centre, as represented by the broken lines in this figure. If the smallest $x$ at which density information is given for the true system is $x_{0}$, then that for the deprojection model will be $x_{1}$, where $x_{1}>x_{0}$, for a given choice of the radial binning. In lieu of any information available for the radial bins inner to $x_{1}$, the code will spread light out uniformly over these bins, using the same density that is recovered at $x_{1}$. Thus, the deprojected density profile will appear to manifest a larger core than the true density profile.

However, when the inequality: $\xi_{\mathrm{D}}>\xi_{\mathrm{T}}$ is stronger at larger than at smaller $x$, the deprojected density profile will end up being flatter on the outside than the true system.

In contrary to this case, if we have a deprojection model that generally corresponds to a lower $\xi$ at any $x, y$ on the POS, than in the true system, the code will attribute a given density to a lower value of $x$ in the density profile recovered with the deprojection model than in the true system. The deprojected density profile will then appear to manifest a similar shape to the true density profile, but will have a comparatively lower amplitude. This case is shown in the right panel of Fig. 2.

In general, we can base our understanding of the density structure in a cluster, by relying on the following ideas that are illustrated in Figs. 2 and 1:

- in two prolate systems with the same long axis, the cluster with the relatively smaller short axes will imply a bigger $\xi$ at a general point, as long as the point in question lies close to the long axis of the system;

- similarly, in two prolate systems with the same short axes, the bigger is the long axis, bigger is $\xi$ to a general point, as long as this comparison is sought within the extent of the smaller of the two long axes in question;

- in two oblate systems with the same long axes, the smaller the short axis, the bigger is $\xi$ to a general point $x, y, z$, (the point is considered a part of both systems); 


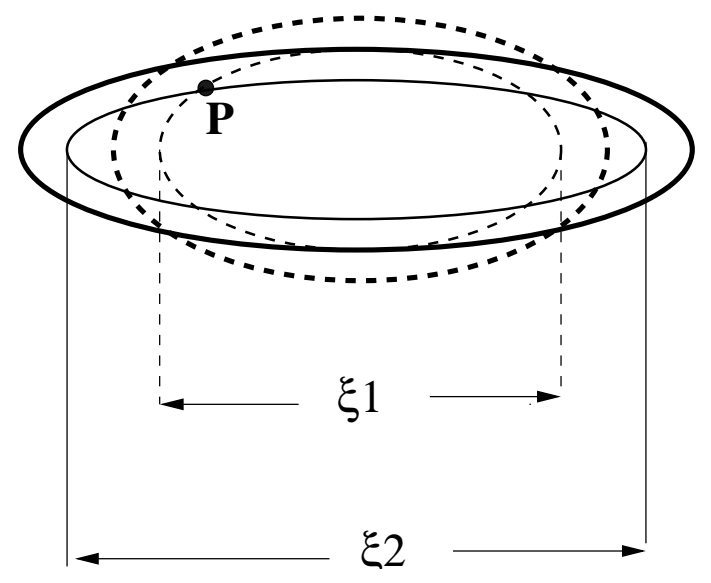

Fig. 1. The ellipsoidal radius to a point $P$ is marked as $\xi 1$ and $\xi 2$, for the two different ellipsoidal configurations, the projection on the plane of the paper of which are shown in thick solid and thick broken lines. The system with the bigger long axis and the smaller short axis corresponds to a higher ellipsoidal radius. Along with the variations in depth, differences in ellipsoidal radius is the other factor that is affected by the choice of the deprojection model.

- similarly, when we consider two oblate systems with the same short axes, the cluster with the bigger long axes corresponds to a bigger $\xi$;

- in general, it is true that a prolate system with the same axial ratio as an oblate system, will imply a larger $\xi$ to the point $x, y, z$ than the oblate system;

- it is also generally true that deviations of an observed cluster from a prolate deprojection model will affect the outer parts of the density profiles while differences between an observed system and an oblate model implies differences in the recovered density profile that will manifest themselves at lower values of $x$.

\subsubsection{True system: $T_{\mathrm{i}}^{\mathrm{p}}$}

Let us elucidate the effects discussed in Sects. 3.2.1 and 3.2.2, in reference to the true system $T_{i}^{\mathrm{p}}$, i.e. a cluster that is prolate in shape and is inclined at a angle $i \mid i_{\min }<i<90^{\circ}$. The projected axial ratio is $q$ and the extent along the photometric semi-major axis is $a$.

A generic $T_{i}^{\mathrm{p}}$ system is shown on the left in Fig. 4; the X-ray $S B$ observed from this true system is then deprojected under the 4 deprojection scenarios that are shown in progression in Fig. 4. In the context of this figure, the truly prolate system is projected along an inclination such that the projected axes on the POS are $b(<a)$ and $a / q$ (by construction of the coordinate systems). If we now assume that this projection on the POS is due to a system that is prolate and inclined at $90^{\circ}$ to the LOS, then that system will be ascribed intrinsic axes of $a / q, a / q \& b$. If however, the system is assumed to be prolate and inclined at an angle $i_{\min }(<i)$, the long axis of the system will have to exceed that of the true system, in order for this configuration to project to the observations. The state of the system, if assumed oblate and inclined at either $90^{\circ}$ or $i_{\min }$, is shown in the second from right and rightmost positions in Fig. 4.

Thus, we see that given a $2 \mathrm{D}$ image, it is possible to guess the semi-axes lengths of the 4 systems that can be described by the 4 different $D$ that we use herein. Now the question arises as to how the run of $\rho$ with $x$ is dictated by the lengths of the semiaxes in the corresponding configuration; more to the point, we
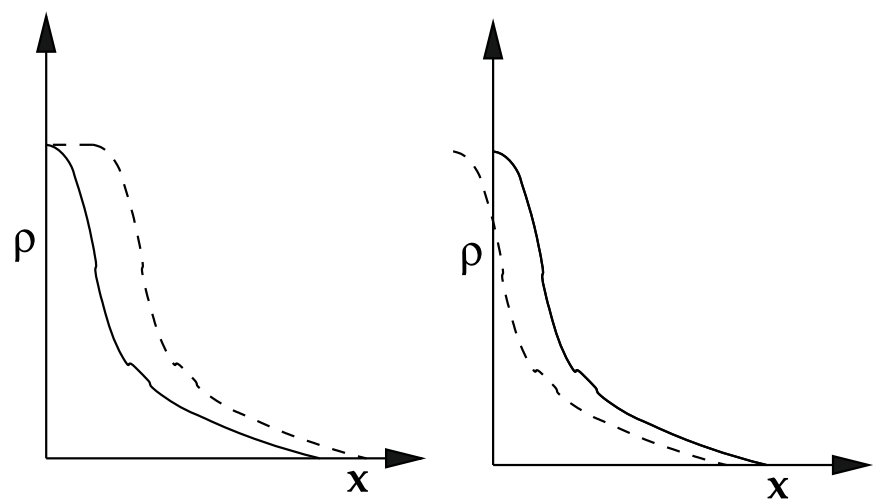

Fig. 2. If the ellipsoidal radius to most points on the $z=0$ plane in the deprojection model is less than that in the true system, then density of the deprojection model will in general be higher than in the true system. In this case, the density profile recovered along the $x$-axis will be characterised by the same value of density appearing at smaller values of $x$. This results in a deprojected density profile that is as shown in broken lines on the right panel. The true density profile of the observed cluster is shown in the same panel, in solid lines. The deprojected profile is shown schematically as simply the amplitude-lessened version of the true profile and therefore is shown to extend to negative $x$-values; in reality, deprojected density values are recovered only for positive $x$. On the other hand, if $\xi_{\mathrm{D}}$ exceeds $\xi_{\mathrm{T}}$ on the average, then deprojected density will fall short of the true density, which will imply a larger core size in the profile recovered in this case (shown in broken lines on the left).

try to gauge how $\rho$ recovered for a given $D$ will compare to the that for another choice of $D$, as well as the true system. The key to such trends lies in the mutual weighing of two configurations for their LOS extents and the ellipsoidal radii to a generic point.

For instance, given that the long axis of $D_{90}^{\mathrm{p}}$ is shorter than that of $T_{i}^{\mathrm{p}}$, we realise that the ellipsoidal radius to a point is higher in $T_{i}^{\mathrm{p}}$ than in $D_{90}^{\mathrm{p}}$ (2nd itemised point in the last list in Sect. 3.2.2). Thus, the density profile recovered by deprojecting under $D_{90}^{\mathrm{p}}$ will have a similar shape as the true $\rho$, but will be lower in amplitude. However, the details of the 3 semiaxes lengths of the $D_{90}^{\mathrm{p}}$ suggests that the LOS extent is lower in $D_{90}^{\mathrm{p}}$ than in $T_{i}^{\mathrm{p}}$. Thus, the lessening of the amplitude caused by differences in $\xi$ is compensated by differences in LOS extent. Similarly, we can track how the $\rho$ recovered for the other $3 D$ will tally with the true density profile, as well as how these recovered profiles compare with each other. The details of the other cases are listed below. We then register such an intercomparison as possible, when the true system is $T_{i}^{\mathrm{p}}$, with $i \in\left(i_{\min }, 90^{\circ}\right)$. Thus, whenever the $S B$ of an observed system, when deprojected under the 4 used $D$ s, correspond to $\rho$ profiles that all have the same shape and the amplitudes of which tally in the way described below, we turn our experience around and declare the system to be prolate in nature with inclination $<i_{\min }$.

Had the inclination of the true system at hand $\left(T_{i}^{\mathrm{p}}\right)$ been such that $i<i_{\min }$, then even for $D_{\min }^{\mathrm{p}}$, the long-axis would have fallen short of the long axis of the true system, as for the $D_{90}^{\mathrm{p}}$ case. In this case, the recovered profile of $\rho$ would have compared with the true density profile similar to the $\rho$ recovered for $D_{90}^{\mathrm{p}}$. Thus, the intercomparison of the density profiles recovered in this case is registered as possible when the true system is $T_{i}^{\mathrm{p}}$, with $i \in\left(0, i_{\min }\right)$.

We qualify the word "possible" in the two instances above, simply because we are finding a possible answer to the question of: what is the correct shape and inclination of the cluster that 
projects to the observations. In other words, we do not claim that our answer is unique. It maybe possible in some circumstances, to conjure up a different geometry+inclination specification, that will correspond to the same projection. We discuss this in Sect. 7.

Had we used other deprojection configurations, the intrinsic axial ratios in such configurations would have been different from what they are for the $4 D$ that we use. The intercomparison of the profiles for $\rho$ that emanate from such conjured configurations (the $D$ of our choice), is rendered easier to interpret given that the $D$ of our choice correspond to the extrema of the geometry and inclination scales.

Some details:

- intrinsic axial ratio of this true system is higher than the deprojection models inclined at $90^{\circ}$;

- with the intrinsic major-axis exceeding that of the $D_{90}^{\mathrm{p}}$ case but the intrinsic minor-axis exceeding that of the $D_{\min }^{\mathrm{o}}$ model;

- the intrinsic major axis of the true system falls short of the same in the $D_{\min }^{\mathrm{p}}$ but the intrinsic minor axis falls short of that in the $D_{90}^{\mathrm{o}}$ model.

For the 4 different deprojection models, the characteristics are enumerated as follows:

1. $D_{90}^{\mathrm{p}}$ :

- $\operatorname{LOS}_{\mathrm{D}}<\mathrm{LOS}_{\mathrm{T}} \Longrightarrow$ amplitude of $\rho_{\mathrm{D}}$ at the centre exceeds that of $\rho_{\mathrm{T}}$;

- ellipsoidal radius to a general point $x, y, z$ is $\xi_{\mathrm{D}}<\xi_{\mathrm{T}} \Longrightarrow$ $\rho_{\mathrm{D}}>\rho_{\mathrm{T}}$;

- this results in deprojected density profile $\left(\rho_{\mathrm{D}}^{x}\right)$ showing a similar shape as $\rho_{\mathrm{T}}^{x}$, but is lower in amplitude.

2. $D_{\min }^{\mathrm{p}}$ :

m ${ }^{\min }{ }^{\circ} S_{\mathrm{D}}>\operatorname{LOS}_{\mathrm{T}} \Longrightarrow$ amplitude of $\rho_{\mathrm{D}}$ falls short of $\rho_{\mathrm{T}}$;

$-\xi_{\mathrm{D}}>\xi_{\mathrm{T}}$;

- the density comparison elicited by this inequality implies that on the outside, $\rho_{\mathrm{D}}^{x}$ is lower in amplitude and is also flatter than $\rho_{\mathrm{T}}^{x}$. This flatness will be less than that for a similarly eccentric, true oblate system, for this deprojection scenario (see next sub-section).

3. $D_{90}^{\mathrm{o}}$

- $\operatorname{LOS}_{\mathrm{D}}=a$, which is in excess of $\operatorname{LOS}_{\mathrm{T}} \Longrightarrow$ amplitude of $\rho_{\mathrm{D}}$ falls short of that of $\rho_{\mathrm{T}}$;

$-\xi_{\mathrm{D}}<\xi_{\mathrm{T}}$

- this implies that $\rho_{\mathrm{D}}^{x}$ shows up with a shape that is similar to the true system but is lower in amplitude; in fact the recovered profile is similar to that recovered from $D_{\min }^{\mathrm{p}}$.

4. $D_{\min }^{\mathrm{o}}$ :

$-\operatorname{LOS}_{\mathrm{D}}<\operatorname{LOS}_{\mathrm{T}} \Longrightarrow$ amplitude of $\rho_{\mathrm{D}}>\rho_{\mathrm{T}}$;

- given that this case corresponds to a smaller minor axis than the true system, and that the true system is described by a comparatively larger intrinsic major axis, $\xi_{\mathrm{D}} \approx \xi_{\mathrm{T}}$ for locations that overlap between the true system and deprojection model.

- $\rho_{x}\left(D_{90}^{\mathrm{o}}\right)$ has similar shape as the true system and is highest in amplitude out of the 4 deprojection models.

\subsubsection{True system $T_{i}^{\circ}$}

Following similar logic as used in the last section, we conclude that when the observed cluster is oblate, inclined at an angle $i \mid i_{\min }<i<90^{\circ}$, the density profiles recovered from the 4 deprojection models are as follows.
- $D_{90}^{\mathrm{p}}$ : recovered density profile will have similar shape as the true system but will be higher in amplitude from LOS considerations.

- $D_{\min }^{\mathrm{p}}$ : recovered density profile will manifest a larger core than the true profile and higher in amplitude from LOS considerations.

- $D_{90}^{\text {o }}$ : similar shape to true profile but amplitude will be lower from considerations of ellipsoidal radius but can be either lower or higher than the true profile, from LOS considerations.

- $D_{\min }^{\mathrm{o}}$ : recovered profile will have the same shape and higher amplitude.

\subsubsection{Triaxial systems}

Here are some types of triaxial systems that we can identify via the inter-comparison of the deprojected luminosity density profiles in our sample clusters. This is discussed in terms of the extent along the principal axis that is identified as the LOS for an inclination of $90^{\circ}$ - referred to as the $Y$-principal axis in the following discussion.

- A triaxial configuration that deviates from a perfectly oblate shape in that the $Y$-principal axis is the longest of the three while the principal axis that corresponds to a photometric axis being the second longest - this is referred to as "triaxialtype I" (left-most panel in Fig. 3).

- It is also possible that the true system is triaxial in the same way as type I systems but the extent along the $X$-axis is only slightly smaller than that along $Y$, so that for inclinations close to $90^{\circ}$, the photometric semi-axes in the $x$-direction exceeds that along the $y$-direction, in contrast to the POS picture for type I systems. Such a triaxial configuration is referred to as "triaxial-type II" (middle panel in Fig. 3).

- If the sole deviation from pure prolateness that is responsible for triaxiality is such that the $Y$-principal axis is the shortest of the three, while the principal axis on the POS has intermediate extent, then the ensuing triaxial configuration is referred to as "triaxial-type III" (right panel of Fig. 3).

- Since the relation between the projected and intrinsic axial ratios in the triaxial case is different from that of the oblate and prolate cases, it is not possible to extract accurate constraints on the system inclination from the deprojection work alone. However, in lieu of any other information, we can make an approximate guess about what the inclination range for a certain triaxial cluster is. This is done by suggesting if the inclination is intermediate to $90^{\circ}$ and $i_{\min }$ or less than $i_{\min }$. The correct estimate of the allowed inclination range for a triaxial cluster will be made on the basis of the pair of relations (that are discussed below), between the projected and intrinsic axial ratios of the triaxial system.

\subsubsection{Other important predictions}

We can pursue logic similar to what was used above, to arrive at the following, critically important conclusions.

- When the true system is $T_{90}^{\mathrm{p}}$, all profiles will be similar in shape, with the relative amplitudes of the profiles recovered under the 4 different deprojection scenarios, as for a truly prolate system, inclined at a general angle $i$.

- When the true system is $T_{90}^{\mathrm{o}}$, all profiles will be similar, with the amplitudes as in the case of inclination at a general angle $i$. 

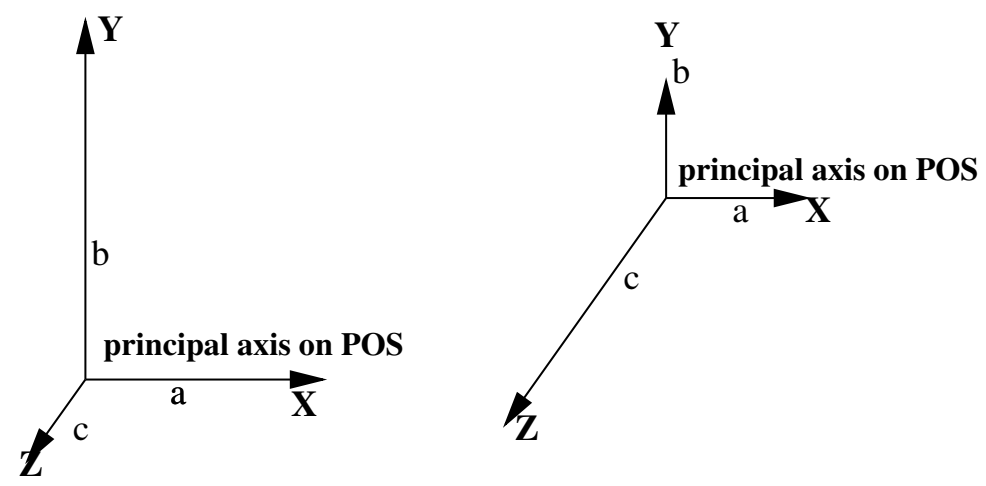

Fig. 3. The three kinds of triaxial systems encountered in this work - types I and II (left, $b>a>c)$ and type III (right, $b<a<c)$. The high inclination and low $b / a$ ratio in type II systems ensures that the photometric major axis is along the $x$-direction, unlike in type I systems.

- For a truly prolate cluster, the deprojected profiles will be further apart, the smaller the angle of inclination is. On the other hand, for a truly oblate cluster, the amplitude differences between the recovered density profiles will increase with decreasing inclination.

\section{Tests}

Here, we report the tests that were carried out to confirm the predictions that were discussed in the last section. These tests were performed with 3 clusters that are either perfectly prolate or oblate, inclined at an angle intermediate to $90^{\circ}$ and $i_{\min }$, namely $60^{\circ}$. In order to demonstrate the independence of the results from the core radius of the system, 2 of the 3 test models were assigned a core radius of approximately $0.01 \mathrm{arcmin}$ and the other has a core radius of about 0.2 arcmin; all the test clusters are assigned a projected axial ratio of $q_{\mathrm{p}}=1.2$. Surface brightness data for each of these test models were estimated by performing a LOS integration of the X-ray luminosity density distributions that were chosen to admit an analytically integrable form.

Each of the test brightness profiles was deprojected under the 4 extreme deprojection scenarios: $D_{90}^{\mathrm{p}}, D_{\min }^{\mathrm{p}}, D_{90}^{\mathrm{o}}$ and $D_{\min }^{\mathrm{o}}$. The density profile that was recovered by deprojecting the brightness data of any test model, under any deprojection scenario, was reprojected back on the POS and this POS distribution was then compared to the input brightness data, to ensure the acceptability of the test run.

The X-ray luminosity density profiles that were recovered with the different deprojection scenarios, for the 3 different test models, are displayed in Fig. 5. The similarity of the intercomparison of the 4 different deprojected density profiles, in the cases of the two prolate clusters with the varying core radii, vindicates the core size independence of the shape determination. However, is this an acceptable conclusion, in terms of the expanse and details of the test systems we considered?

\subsection{Generality of tests}

In this section we examine the fundamental question of how general our test systems are. To begin with, a statistical confirmation of our method is a far cry from the limited tests that we have presented below. It would be judicious to scan a range of ellipticities and core radii corresponding to the distribution of these quantities, as constrained by observations and simulations. Furthermore, the redshift dependence of these distributions has to be folded in, to test for our shape determination technique (as indicated by the ellipticity-redshift relation). In fact, work is underway to calibrate our technique against a sample of simulated gas+dark matter halos from the Millennium Gas Simulations (Chakrabarty et al. 2008).

While such a rigorous statistical test is on, we have presented here tests done with three clusters that have characteristics similar to what have been noted with the sample of clusters that we apply our method to (following section) - as apparent from Table 1, in our cluster sample, there are 7 out of 25 clusters, that have a projected axial ratio lying between 1.2 and 1.25 , approximately (including A 399 which is at a redshift of 0.072). Most of the other systems manifest even more eccentric 2D images. In fact, De Filippis et al. (2005) state that the redshift-ellipticity relation is not manifest in this sample. Thus, our test configurations as motivated by measurement.

\section{Application}

We apply our formalism to a sample of 25 clusters from Reese et al. (2002). This sample consists of $18 \mathrm{X}$-ray selected clusters with $z \geq 0.14, \delta \geq-15^{\circ}$ and $L_{X}(0.1-2.4 \mathrm{keV}) \geq 5 \times$ $10^{44} h_{50}^{-2} \mathrm{erg} \mathrm{s}^{-1}$ and for which high S/N detections of SZE, high$\mathrm{S} / \mathrm{N}$ X-ray imaging and electron temperatures were available. To these, 7 clusters from the Mason et al. (2001) sample were added, containing clusters from X-ray flux-limited catalogue of Ebeling et al. (1996). Details on the completeness of the latter subsample are given by Mason \& Myers (2000). Actually, we choose to work with 24 of these 25 clusters since the measured surface brightness profile of one of the sample clusters (A 520) appears to be plagued by very large observational error bars in the last 3 arcmin (typically, in excess of 50\%).

The motivation behind choosing this sample is that the threedimensional intrinsic morphology of the clusters in this sample has already been reported, as estimated by an independent method that combines X-ray and Sunyaev-Zel'dovich observations (Sereno et al. 2006). Thus, we will be in a position to compare our results with that from Sereno et al. (2006).

\subsection{Data analysis}

In our analysis we have used archival Chandra or XMM-Newton data for all clusters in the sample. The Chandra Interactive Analysis of Observation software (CIAO 3.3.0.1) and the XMM-Newton Science Analysis Software (SAS 7.0.0) were used throughout.

We first modelled the projected two dimensional cluster emission, in the $0.3-7.0 \mathrm{keV}$ energy band. Pixel values of all detected point sources were replaced with values interpolated 

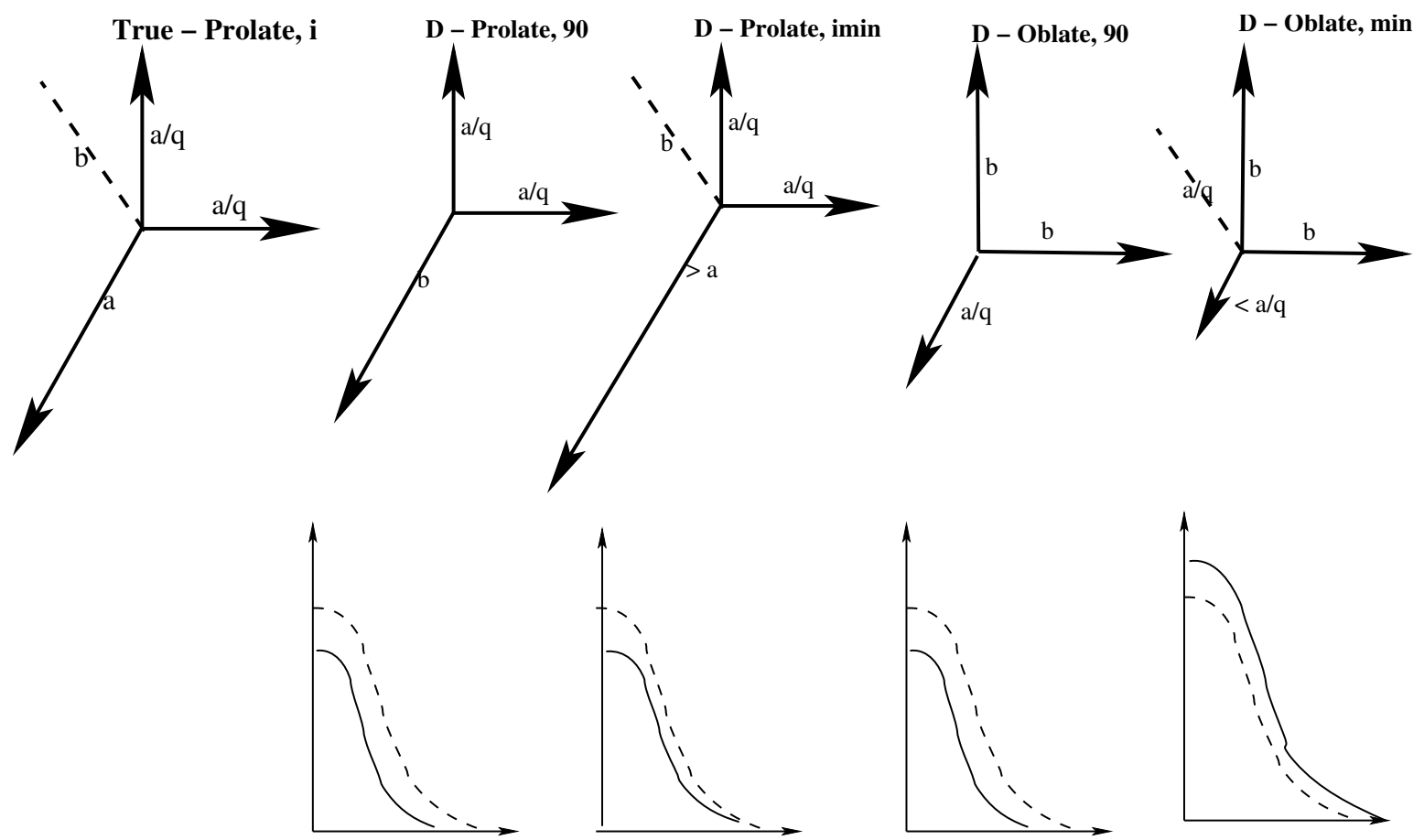

Fig. 4. Figure showing interpretation of the observed prediction of a cluster that is truly prolate and inclined at an angle $i \in\left[0,90^{\circ}\right]$ (shown on the top left-most corner), under 4 deprojection scenarios: $D_{90}^{\mathrm{p}}$ (second from left), $D_{\min }^{\mathrm{p}}$ (third from left), $D_{90}^{\mathrm{o}}\left(\right.$ second from right) and $D_{\min }^{\mathrm{o}}($ right). The cluster is inclined such that its intrinsic axial ratio $q$ results in the POS projected axial ratio of $b: a / q$. The intrinsic semi-axes lengths that need to be ascribed to the 4 different $D$ configurations are: $\xi_{90}^{\mathrm{p}}<\xi^{\mathrm{T}}, \xi_{\min }^{\mathrm{p}}>\xi^{\mathrm{T}}, \xi_{90}^{\mathrm{o}}<\xi^{\mathrm{T}}$ and $\xi_{\min }^{\mathrm{o}} \approx \xi^{\mathrm{T}}$. The comparison in terms of the LOS extent is such that only for $D_{\min }^{\mathrm{p}}$, it is in excess of that in the true system; for the other $3 D$, it falls short of that in the true system. In light of these comparisons, (according to the last bulletted list in Sect. 3.2.2), the recovered deprojected density profile along $x$, is shown in the lower panels (in solid lines), for the corresponding $D$, along with the true density profile, plotted in broken lines.

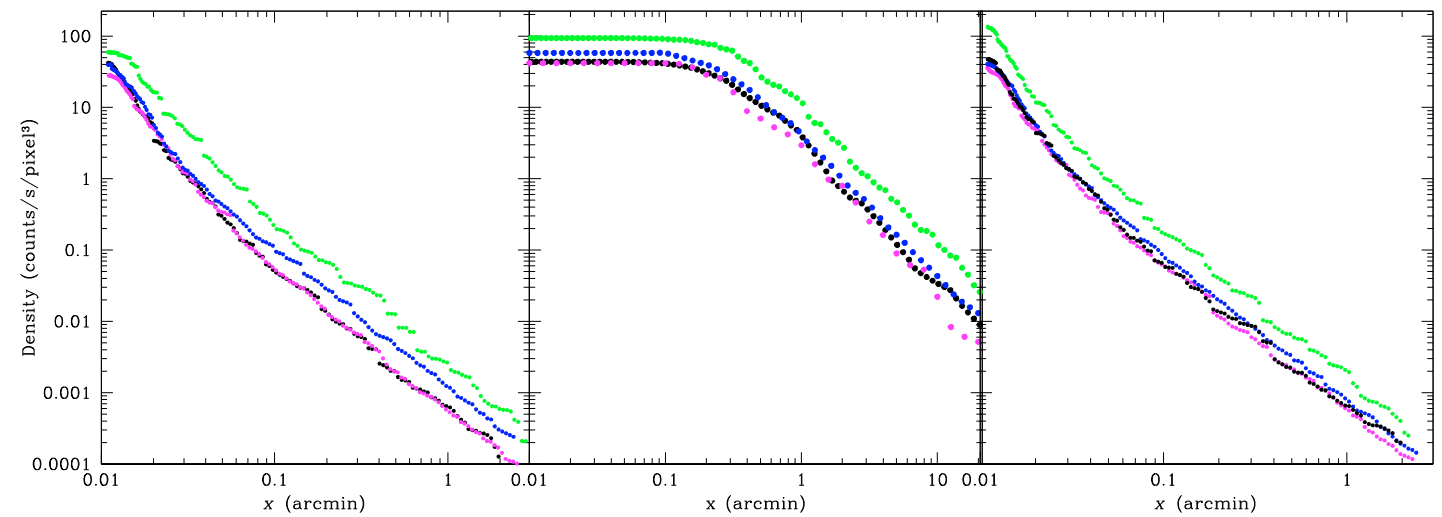

Fig. 5. X-ray luminosity density profiles of different test clusters, recovered by deprojecting the toy X-ray surface brightness maps of these systems. The test systems include prolate (right and middle) and oblate (left) models with core size of about 0.003 arcmin (left and right) and 0.2 arcmin (middle); the projected axial ratio is 1.2 for all three test systems. The density profiles recovered under the $D_{90}^{\mathrm{p}}$ scenario is in magenta, the $D_{\min }^{\mathrm{p}}$ case in blue, $D_{90}^{\mathrm{o}}$ in black and $D_{\min }^{\mathrm{o}}$ in green.

from the surrounding background regions. Using the SHERPA software, we fitted the cluster surface brightness to elliptical 2D $\beta$-models. The resulting best fit parameters for all clusters in the sample, relevant for this paper, are listed in Table 1.

We then extracted one dimensional surface brightness profiles within elliptical concentric annuli, with projected ellipticity, position angle and centre matching those estimated in the our 2D analysis (as listed in Table 1). The distance $x$ from the cluster centre is measured along the major axis of the ellipses. The profiles were extracted in the $0.3-7.0 \mathrm{keV}$ energy range. In the same energy band, the background for each cluster was estimated, within a peripheral region of the field of view. Corresponding radial profiles, extracted within the same elliptical regions from the exposure maps, provided the required average values of the effective exposure time within each annulus. Background subtracted and exposure corrected surface brightness profiles were then extracted for each cluster.

\section{Results}

The intrinsic shapes and inclination classes that we infer for our chosen sample of clusters are enumerated in Table 2. We also include the shapes and inclinations of the clusters, as predicted by Sereno et al. (2006), in adjacent columns. 
Table 1. Cluster sample.

\begin{tabular}{lcccccc}
\hline \hline & & & $x_{\mathrm{c}}, y_{\mathrm{c}}$ & & & \\
& & RA & Dec & $q$ & $\begin{array}{c}\theta \\
\text { deg }\end{array}$ & satellite \\
\hline MS 1137.5+6625 & 0.784 & 114022.3 & +660815.3 & $1.113 \pm 0.014$ & $116.1 \pm 1.0$ & 1 \\
MS 0451.6-0305 & 0.550 & 045411.4 & -030051.3 & $1.307 \pm 0.015$ & $95.9 \pm 1.1$ & 1 \\
Cl 0016+1609 & 0.546 & 001833.5 & +162612.9 & $1.205 \pm 0.013$ & $310.8 \pm 1.7$ & 2 \\
RXJ1347.5-1145 & 0.451 & 134730.7 & -114509.1 & $1.453 \pm 0.019$ & $158.5 \pm 1.0$ & 1 \\
A 370 & 0.374 & 023953.3 & -013439.0 & $1.564 \pm 0.018$ & $6.2 \pm 0.7$ & 1 \\
MS 1358.4+6245 & 0.327 & 135950.7 & +623104.1 & $1.325 \pm 0.019$ & $156.6 \pm 1.4$ & 1 \\
A 1995 & 0.322 & 145257.9 & +580255.8 & $1.242 \pm 0.010$ & $57.8 \pm 1.0$ & 1 \\
A 611 & 0.288 & 080056.8 & +360323.5 & $1.14 \pm 0.05$ & $34 \pm 9$ & 1 \\
A 697 & 0.282 & 084257.6 & +362156.8 & $1.334 \pm 0.016$ & $163.8 \pm 1.2$ & 1 \\
A 1835 & 0.252 & 140102.0 & +025242.9 & $1.225 \pm 0.012$ & $173.0 \pm 1.4$ & 1 \\
A 2261 & 0.224 & 172227.1 & +320757.4 & $1.022 \pm 0.017$ & $90.0 \pm 1.7$ & 1 \\
A 773 & 0.216 & 091753.1 & +514337.9 & $1.237 \pm 0.022$ & $90.0 \pm 2.5$ & 1 \\
A 2163 & 0.202 & 161546.6 & -060844.9 & $1.206 \pm 0.004$ & $90.0 \pm 0.6$ & 1 \\
A 1689 & 0.183 & 131129.6 & -012028.0 & $1.141 \pm 0.012$ & $17.6 \pm 2.2$ & 1 \\
A 665 & 0.182 & 083057.1 & +655101.8 & $1.238 \pm 0.012$ & $146.3 \pm 1.2$ & 1 \\
A 2218 & 0.171 & 163551.9 & +661234.6 & $1.162 \pm 0.009$ & $96.5 \pm 1.5$ & 1 \\
A 1413 & 0.142 & 115517.9 & +232416.2 & $1.473 \pm 0.019$ & $177.8 \pm 0.9$ & 1 \\
A 2142 & 0.091 & 155820.1 & +271403.5 & $1.540 \pm 0.007$ & $127.7 \pm 0.3$ & 1 \\
A 478 & 0.088 & 041325.3 & +102753.5 & $1.477 \pm 0.006$ & $43.5 \pm 0.3$ & 1 \\
A 1651 & 0.084 & 125921.9 & -041144.6 & $1.184 \pm 0.013$ & $87.6 \pm 1.9$ & 1 \\
A 401 & 0.074 & 025857.1 & +133437.8 & $1.303 \pm 0.008$ & $34.9 \pm 0.6$ & 1 \\
A 399 & 0.072 & 025752.0 & +130238.7 & $1.207 \pm 0.009$ & $22.5 \pm 1.2$ & 2 \\
A 2256 & 0.058 & 170400.4 & +783837.1 & $1.327 \pm 0.008$ & $28.7 \pm 0.5$ & 2 \\
A 1656 & 0.023 & 125944.1 & +275643.0 & $1.141 \pm 0.006$ & $90.0 \pm 0.6$ & 2 \\
\hline
\end{tabular}

Column 1: cluster name, Col. 2: cluster redshift, Cols. 3-6: fit parameters of the elliptical 2D $\beta$ model: $x_{\mathrm{c}}, y_{\mathrm{c}}$ is the central position; $q$ is the projected axial ratio, and $\theta$ is the orientation angle (north over east). In the last column, label 1 is for Chandra and 2 for XMM observations.

Thus, we find that in our sample of 24 clusters, 15 are triaxial, 1 is oblate and the rest (8) are prolate. This shape distribution is significantly different from that deciphered by Sereno et al. (2006), as evident in Table 2; we discuss the differences in detail in Sect. 7.1.

We also looked at the central flux distribution for the clusters in our sample and sought correlations with the recovered shapes; in particular, we attempted to establish is any correlation exists between the clusters and the central flux measurements. This was found to be absent.

\subsection{Incorporation of SZe data to prolate and oblate clusters}

The impending improvement in the quality of the SZe data will boost this technique greatly. Knowledge of the extent along the LOS will permit us to perform the deprojection in a fully triaxial geometry - such a deprojection is possible with DOPING. Thus, while in the current version of DOPING we compare the planeof-the-sky projection of the recovered density distribution to the observed brightness map of the system, on improvement of the SZe data, the LOS extent of the density structure can also be pinned down, for a given choice of the inclination. We would then be in a position to better constrain both the intrinsic axial ratios for the triaxial case and thereby improve upon the estimate of the inclination of the cluster to the LOS.

As mentioned above, one motivation for choosing this sample is the availability of the SZe data for all the clusters in the sample. This was reported by De Filippis et al. (2005). It is not surprising that the ratio of the LOS extent to the photometric major axis $\left(q_{\text {LOS }}\right)$ of a sample cluster is characterised by large error bars.

We implement the reported $q_{\text {LOS }}$ first for all the clusters that are recovered as prolate in our sample, and then for the oblate ones. To do this, we use the following relations (from Sereno et al. 2006):

$$
\begin{aligned}
q_{\mathrm{LOS}} & =\frac{\sqrt{q_{\mathrm{p}}^{2}-\cos ^{2} i}}{q_{\mathrm{p}}^{2} \sin i} \quad \text { prolate } \\
& =\frac{\sqrt{1-q_{\mathrm{p}}^{2} \cos ^{2} i}}{\sin i} \quad \text { oblate }
\end{aligned}
$$

The reported $q_{\mathrm{p}}$ and $q_{\mathrm{LOS}}$ values are used in this equation, for a sample cluster that has been identified as prolate. The values of $q_{\mathrm{LOS}}$ reported at the two extrema of the error bands, for any cluster, results in a range for the inclination. These inferred range on the inclination is superimposed on the range of inclinations that were judged admissible from deprojection considerations alone. The range corresponding to the overlap of these two sets of constraints is then narrower, as represented in the last column of Table 3. A similar exercise was undertaken for the sole oblate cluster in our sample (according to our shape evaluation); the improved inclination estimate for this system is included as the last entry in Table 3.

As can be appreciated from this table, the inclusion of SZe data makes a significant effect in constraining the cluster inclination. However, the allowed inclination ranges from the X-ray data and the SZe data did not overlap for the case of Abell 611; thus, we are unable to produce an inclination for this cluster.

\subsection{Incorporation of SZe data to triaxial clusters}

The direct exploitation of the SZe data is possible only for the cases of the purely oblate or purely prolate clusters. For the 

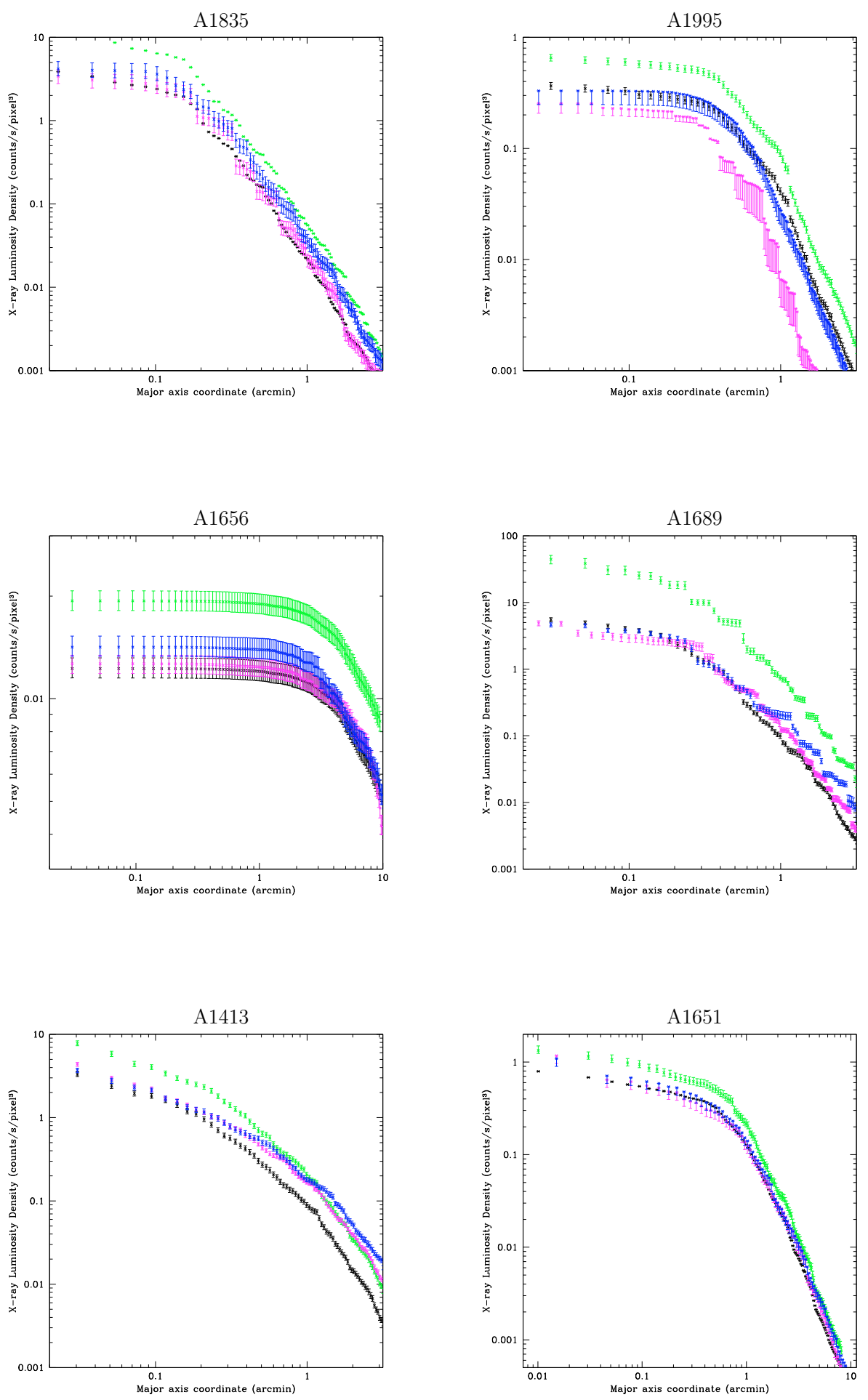

Fig. 6. X-ray luminosity density profiles of 6 of the 24 clusters in our sample. The colour scheme used for the profiles recovered under the 4 different deprojection scenarios is the same as in Fig. 5.

general triaxial clusters, the SZe information is used to process the two intrinsic axial ratios, given that we know how the lengths of the three semi-axes compare, given the recovered triaxiality type. Additionally, the cluster inclination can be pinned down from the recovered inequalities that relate the intrinsic axial ratios to unity. Such constraints too are derived from the identification of the type of triaxiality in question. The details of the methodology used to constrain axial ratios and inclinations is delineated in Appendix A.
As mentioned above, the inclinations guessed from the X-ray deprojection exercise alone are loose approximations only and only the values of $i$ derived from the incorporation of the SZe data should be considered correct.

The axial ratios in the considered triaxial cases are tabulated, along with the improved inclination constraints, in Table 4. It is to be noted that these constraints are estimated by ignoring the errors that are reported (De Filippis et al. 2005) on the elongation of the sample clusters. The axial ratios are calculated with the 
Table 2. Shapes and inclinations of cluster sample.

\begin{tabular}{|c|c|c|c|c|}
\hline & Inferred shape & $\begin{array}{c}\text { Approximate Incl } \\
\text { (degrees) }\end{array}$ & Shape in Sereno et al. (2006) & $\begin{array}{c}\text { Incl in Sereno et al. (2006) } \\
\text { (degrees) }\end{array}$ \\
\hline MS $1137.5+6625$ & prolate & $26<i<90$ & prolate & $16 \pm 9$ \\
\hline MS 0451.6-0305 & triaxial-type I & $40<i<90$ & prolate/oblate & $56 \pm 13 / 58 \pm 43$ \\
\hline $\mathrm{Cl} 0016+1609$ & triaxial-type I & $34<i<90$ & prolate & $26 \pm 10$ \\
\hline RXJ1347.5-1145 & triaxial-type III & $i<47$ & oblate/prolate & $84 \pm 117 / 35 \pm 12$ \\
\hline A 370 & triaxial-type II & $i<50$ & prolate & $27 \pm 12$ \\
\hline MS 1358.4+6245 & prolate & $i<41$ & oblate & $52 \pm 8$ \\
\hline A 1995 & triaxial-type III & $i<36$ & oblate/prolate & $64 \pm 35 / 46 \pm 27$ \\
\hline A 611 & prolate & $29<i<90$ & prolate & $35 \pm 32$ \\
\hline A 697 & triaxial-type I & $42<i<90$ & prolate & $26 \pm 10$ \\
\hline A 1835 & prolate & $35<i<90$ & prolate & $29 \pm 7$ \\
\hline A 2261 & prolate & $i<12$ & prolate & $10 \pm 6$ \\
\hline A 773 & triaxial-type II & $i<36$ & prolate & $14 \pm 5$ \\
\hline A 2163 & triaxial-type I & $34<i<90$ & prolate & $28 \pm 10$ \\
\hline A 1689 & prolate & $29<i<90$ & oblate/prolate & $65 \pm 44 / 46 \pm 23$ \\
\hline A 665 & triaxial-type I & $36<i<90$ & oblate & $47 \pm 13$ \\
\hline A 2218 & triaxial-type I & $34<i<90$ & prolate & $22 \pm 11$ \\
\hline A 1413 & triaxial-type II & $i<47$ & oblate/prolate & $73 \pm 33 / 37 \pm 15$ \\
\hline A 2142 & triaxial-type II & $i<50$ & oblate/prolate & $79 \pm 42 / 34 \pm 10$ \\
\hline A 478 & triaxial-type II & $i<48$ & prolate & $23 \pm 12$ \\
\hline A 1651 & prolate & $i<32$ & prolate & $12 \pm 7$ \\
\hline A 401 & triaxial-type II & $i<40$ & prolate & $25 \pm 6$ \\
\hline A 399 & oblate & $34<i<90$ & oblate & $38 \pm 5$ \\
\hline A 2256 & triaxial-type II & $i<41$ & prolate & $34 \pm 15$ \\
\hline A 1656 & prolate & $i<29$ & prolate & $33 \pm 30$ \\
\hline
\end{tabular}

Column 1: cluster name, Col. 2: intrinsic cluster shape recovered from our work, Col. 3: recovered inclination class - in degrees, Col. 4: shape reported by Sereno et al. (2006) and Col. 5: inclination as reported by Sereno et al. (2006) (in degrees) - the clusters for which both prolate and oblate solutions were found admissible by Sereno et al. (2006) are marked accordingly in Col. 4 and the corresponding inferred inclinations are shown in Col. 5.

Table 3. Improved inclinations (using SZe data) of the prolate and oblate sample clusters.

\begin{tabular}{lcccc}
\hline \hline & Inferred shape & $\begin{array}{c}\text { Incl from deprojection } \\
\text { (degrees) }\end{array}$ & $\begin{array}{c}\text { from SZe data } \\
\text { (degrees) }\end{array}$ & $\begin{array}{c}\text { Refined Incl } \\
\text { (degrees) }\end{array}$ \\
\hline MS 1137.5+6625 & prolate & $26<i<90$ & $11<i<63$ & $26<i<63$ \\
MS 1358.4+6245 & prolate & $i<41$ & $0<i<44$ & $41<i<44$ \\
A 61 & prolate & $29<i<90$ & $0<i<20.665$ & - \\
A 2261 & prolate & $i<12$ & $7<i<20$ & $7<i<12$ \\
A 1689 & prolate & $29<i<90$ & $0<i<33$ & $29<i<33$ \\
A 1651 & prolate & $i<32$ & $8<i<32$ & $8<i<32$ \\
A 1656 & prolate & $i<29$ & $19<i<34$ & $19<i<29$ \\
A 1835 & prolate & $35<i<90$ & $13<i<78$ & $35<i<78$ \\
A 399 & oblate & $34<i<90$ & $34<i<37$ & $34<i<37$ \\
\hline
\end{tabular}

Column 1: cluster name, Col. 2: intrinsic cluster shape recovered from our work, Col. 3: inclination recovered from the deprojection exercise, Col. 4: inclination as indicated by relating the projected POS axial ratio and LOS extent (De Filippis et al. 2005), Col. 5: the most improved bounds on inclination, obtained as overlap of constraints imposed by the deprojection exercise and the SZe data.

inclination set to the median of the band of inclinations that are deemed suitable for a given cluster.

\section{Discussions}

In this work we have presented a simple but novel technique to extract the intrinsic shape and inclination class of galaxy clusters, using X-ray brightness maps alone. The availability of SZe data is then shown to improve the inclination estimate considerably. The main motivation behind this exercise is the improvement on mass estimates which is expected to refine the constraints that we can place on cosmological constants, from the analysis of cluster data.
Our suggested formalism relies upon the inter-comparison of the density profiles that are deprojected from the measured X-ray surface brightness maps, under 4 different deprojection scenarios that combine the extrema of the inclination scale and the geometry scale, when clusters are treated as figures of revolution. It is found that each type of observed system leaves its unique signature in this inter-comparison amongst the deprojected X-ray luminosity density profiles. The knowledge of the cluster elongation along the LOS (from SZe data) is then used to supplement our shape and inclination determination; in particular, the recovered constraints on the inclination are significantly improved, if we believe the SZe measurements. In case of triaxial systems, the intrinsic axial ratios can also be tracked, using the SZe data. 
Table 4. Axial ratios and improved inclinations (using SZe data) of the triaxial sample clusters.

\begin{tabular}{lccccc}
\hline \hline & Shape from deprojection & $\begin{array}{c}\text { Approx Incl from Deproj } \\
\text { (degrees) }\end{array}$ & $\begin{array}{c}\text { Correct Incl } \\
\text { (degrees) }\end{array}$ & $q_{1}$ & $q_{2}$ \\
\hline MS 0451.6-0305 & triaxial-type I & $40<i<90$ & $67<i<90$ & 0.92 & 1.34 \\
Cl 0016+1609 & triaxial-type I & $34<i<90$ & $73<i<90$ & 0.65 & 1.24 \\
A 697 & triaxial-type I & $42<i<90$ & $77<i<90$ & 0.58 & 1.33 \\
A 2163 & triaxial-type I & $34<i<90$ & $72<i<90$ & 0.68 & 1.24 \\
A 665* & triaxial-type I & $36<i<90$ & $70<i<90$ & 0.78 & 1.27 \\
A 2218 & triaxial-type I & $34<i<90$ & $78<i<90$ & 0.45 & 1.20 \\
A 370 & triaxial-type II & $50<i<90$ & $73<i<90$ & 0.84 & 1.62 \\
A 773 & triaxial-type II & $36<i<90$ & $78<i<90$ & 0.48 & 1.28 \\
A 1413 & triaxial-type II & $47<i<90$ & $66<i<71$ & 0.96 & 1.64 \\
A 2142 & triaxial-type II & $50<i<90$ & $69<i<77$ & 0.96 & 1.67 \\
A 478 & triaxial-type II & $48<i<90$ & $75<i<90$ & 0.73 & 1.52 \\
A 401 & triaxial-type II & $40<i<90$ & $72<i<90$ & 0.76 & 1.34 \\
A 2256 & triaxial-type II & $41<i<90$ & $67<i<90$ & 0.94 & 1.36 \\
A 1995 & triaxial-type III & $i<36$ & $0<i<31$ & 3.94 & 0.34 \\
RXJ1347.5-1145 & triaxial-type III & $i<47$ & $0<i<65$ & 5.14 & 0.29 \\
\hline
\end{tabular}

Column 1: cluster name, Col. 2: intrinsic cluster shape recovered from our work, Col. 3: approximate inclination range, guessed from the deprojection exercise, Col. 4: bounds on inclination, obtained from the constraints on the intrinsic axial ratios, as indicated by the type of triaxiality (types I, II or III) that is assigned to the cluster from deprojection work, Col. 5: ratio $q_{1}$ between the length of the principal axis $(a)$ that corresponds to one of the photometric axes and that along the $Y$-principal axis (b, see Fig. 3), Col. 6: ratio $\left(q_{2}\right)$ between $a$ and and the other remaining principal axis $(c)$.

${ }^{*}$ For this cluster, the $q_{\mathrm{LOS}}$ value reported at the higher end of the range is used; the medial value of $q_{\mathrm{LOS}}$ constrains the cluster inclination to less than a degree.

\subsection{Differences with results of Sereno et al. (2006)}

Our shape determination agrees with the shape advanced by Sereno et al. (2006), for 13 of the 24 sample clusters. This quantification includes those cases that we identify as triaxial and Sereno et al. (2006) find compatible with both the prolate and oblate geometries. For another 10 cases, while we find the system to be definitely triaxial, (Sereno et al. 2006) report the system to be prolate. This might result from the fact that Sereno et al. (2006) use the observed values of projected axial ratio and LOS extent, in equations relating these quantities and the intrinsic axial ratio to inclination $i$, for the two geometries of prolateness and oblateness (see Eqs. (8)-(11) in Sereno et al. 2006).

They constrain their choice of geometry by identifying realistic solutions for inclinations, such as $-1 \leq \cos (i) \leq 1$. Thus, if for example, they identify an unrealistic solution for $i$ for the oblate case, they declare the system prolate. But strictly speaking, all that they can conclude is that the cluster at hand is not oblate. In other words, it is possible that the cluster is triaxial, in such a way that given the high measurement errors, the equation for $i$ in the prolate case also yields acceptable values. The 10 sample clusters that we spot as triaxial, but Sereno et al. (2006) call prolate, are similarly 10 non-oblate clusters.

The one system that we identify as prolate and Sereno et al. (2006) call oblate i.e. non-prolate, is MS 1358.4+6245. However, when we use the values of 1.325 for the projected axial ratio (ignoring the $\pm 1 \%$ error) and 0.91 for $e_{\mathrm{LOS}}$ (using the upper bound from the measured range of $0.72 \pm 0.19$ ), in Eqs. (8) and (9), we do actually get a realistic inclination of about $44^{\circ}$, under the assumption of prolateness. In other words, the methodology used by Sereno et al. (2006), does not rule out prolateness for this cluster, in line with our inference.

Thus, our methodology is relatively more powerful, since we have greater resolution ability than simply ruling out prolateness/oblateness. Moreover, our implementation of the SZe data allows for the recovery of a much narrower range of inclinations, for the same measurement as used by previous workers.

\subsection{X-rays vs. Optical SB}

We would like to emphasise that it is very much possible to utilise the optical surface brightness distribution of clusters, if available, to make an estimate of the morphological structure of the cluster with the aid of DOPING. This would allow the verification and quantification of possible discrepancies between results obtained using X-ray and optical data. This is particularly interesting, given the study presented by Gottlöber \& Yepes (2007) which suggests a relatively more spherical central gas component, compared to the dark matter one.

To this aim, we are now applying the DOPING algorithm to both dark matter halos from the Millennium Simulations and to gas+dark matter $S B$ distribution of the same halos (from the Millennium Gas Simulations). Results will be published in Chakrabarty et al. (2008). This will answer the question of how the shape determination is affected by changes in the nature of the input.

In this connection, it is further important to mention that our sample of real clusters show more eccentric gas distribution in projection, than the simulated ones studied in the hydrodynamics simulations of Gottlöber \& Yepes (2007) and, consequently, the intrinsic axial ratios that we recover, are not akin to $q_{1}=1, q_{2}=$ 2 , as suggested in that work.

\subsection{Core sizes}

We tested our predictions on a suite of model clusters and found that the recovery of shapes and inclinations is independent of the axial ratios that were assigned to these test systems, as well as the core sizes that these test systems were described by. That the core size is not influential in the recovery of the intrinsic shape of the cluster is not surprising since it is the outer part of the brightness profile that is conventionally considered when identifying shapes of clusters. Thus, whether it is Abell 2261 or MS 1358, our formalism is able to do justice to systems at both ends of core sizes in the sample used herein. This brings us to an 
important point in regard to the applicability of our technique it can be implemented to estimate the 3D geometry of clusters at varying redshifts! In fact, the sample that we used to illustrate the efficacy of our formalism is rather eclectic in its redshift coverage, $(z=0.023$ to 0.784$)$.

Crucially, our work has clearly indicated the dependence of the recovered core size of the deprojected luminosity density profile, on the deprojection scenario used. Thus, for Abell 665, the deprojected density corresponding to an assumption of oblateness and $i=i_{\text {min }}$ implies a core size that is less than half $(\approx 0.06 \mathrm{arcmin})$ that obtained by deprojection performed under prolateness and $i=i_{\min }$ and inclination combinations $(\approx 0.15$ arcmin).

It is important to stress here that we do not need to delete the cores from our analysis due to any conceived inability of our code to deal with the modelling of the very central regions of the clusters. The code used in the work is in fact non-parametric and its functionality is not challenged by local changes in slopes of the observed X-ray brightness profiles or even by local enhancements of observational errors in the brightness distributions. The deprojected profile will of course bear larger error bars when the input brightness profile manifests the same, than otherwise. The fact that the inclusion of the core does not affect the shape determination is brought home by the tests that we have performed by varying the core sizes of toy clusters.

\subsection{Central density}

One ramification of the observed nature of the luminositytemperature relationship in clusters is the need to invoke some (unknown) pressure that is effective in reducing the density of the intracluster gas in the cores (Voit et al. 2002). However, as we have seen in this work, the X-ray luminosity density at the centres of clusters depends critically on the deprojection scenario adopted in the model. Thus, in Abell 1689, we notice that the density recovered under an assumption of prolateness and $i=i_{m} i n$, at about 3 arcmin, is about 10 times less than that recovered under the other three deprojection scenarios. Thus, our work indicates the important contribution that deprojection uncertainties can make towards the observed trends in the cluster self-similar relationships.

\subsection{Effect of errors of cluster elongation}

The current state of affairs regarding the quality of SZe data is indeed unsatisfactory, albeit improvements are impending. Thus, when we use the SZe data in this work, we make the conscious decision to work with the value of the cluster elongation that is reported at the centre of the error band. Incorporation of the measurement errors would have greatly reduced the quality of the constraints on the inclinations.

\subsection{The case of Abell 1995 and RXJ1347}

The clusters Abell 1995 and RXJ1347 have been found to be triaxial, of type III, which implies that these clusters are nearly prolate except that $c<a$; also, we expect $b-a \ll a$. The density profiles recovered under the assumption of prolateness are flatter for these clusters than the profiles deprojected under oblateness. This comparison is allowed if the ellipsoidal radius in the prolate cases, in general, exceed that in the oblate cases. This is in turn ensured if $a$ is the photometric semi-axis and if the inclination is small. We find that the inclination is indeed constrained to very small angles, given the reported values of $q_{\mathrm{p}}$ and $q_{\mathrm{LOS}}$ (rather the medial value of these quantities within the reported measurement error bands). Thus, these two clusters are deciphered to be nearly face-on systems.

\subsection{The case of Abell 370}

The cluster Abell 370 was reported to bear a "pronounced triaxial morphology" by De Filippis et al. (2005), on the basis of the X-ray and SZe data. However, Sereno et al. (2006) find the prolate solution to be consistent with the observed X-ray and SZe data for this cluster. In contrast to this, we actually find this cluster to be triaxial, with an inclination in excess of $50^{\circ}$.

\subsection{The case of CL $0016+609$}

Hughes \& Birkinshaw (1998) suggested a "reasonable triaxial" morphology for the cluster CL $0016+609$, on the basis of the discrepancy between the Hubble constant values that were estimated from the oblate and prolate models. This suggestion is in line with our conclusion of a triaxial shape for this cluster.

\subsection{Future work}

The identification of the intrinsic shape and inclination of a cluster is a major step in the characterisation of clusters, both in terms of cluster masses and quantification of the contribution of deprojection uncertainties to the observed scatter in the selfsimilar relationships of clusters. These applications are planned in future contributions.

In fact, it is envisaged that the improved understanding of cluster spatial configurations leads to better constraints on cluster masses. If this is supplemented with information of mass distributions from dynamical considerations (using an algorithm such as CHASSIS - Chakrabarty \& Saha 2001), we could potentially place bounds on the distribution of gas that is at hydrostatic equilibrium. Furthermore, using estimation of the mass within a given projected radius from lensing measurements, would constrain the dynamical mass distribution even better, leading to added improvements in cluster characterisation. An exercise in the determination of improvement in the dynamical mass of a lensing galaxy, using such lensing constraints, is currently underway (Chakrabarty et al. 2008).

Acknowledgements. D.C. is supported by a Royal Society Dorothy Hodgkin Fellowship. H.R. would like to acknowledge the Mary Cannell Summer Studentship that made her contribution possible.

\section{Appendix A:}

Given that in our coordinate system, $X=x$, we say:

$(y, z)=\mathbf{R}_{i}(Y, Z)$

where $\mathbf{R}_{i}$ is the rotational matrix corresponding to a rotation through the angle $i$. Let us consider a triaxial system, with intrinsic axial ratios $q_{1}$ and $q_{2}$, i.e. ratios of the maximum extent along the $X$-axis to that along the $Y$-axis is $q_{1}$ and the ratio of the principle axis along the $X$-axis to that along the $Z$-axis is $q_{2}$.

In this triaxial configuration, the square of the ellipsoidal radius is

$$
\begin{aligned}
\xi= & x^{2}+y^{2}\left[q_{1}^{2} \cos ^{2}(i)+q_{2}^{2} \sin ^{2}(i)\right] \\
& +z^{2}\left[q_{1}^{2} \sin ^{2}(i)+q_{2}^{2} \cos ^{2}(i)\right]+y z \sin (2 i)\left(q_{1}^{2}-q_{2}^{2}\right)
\end{aligned}
$$


This implies that the plane-of-the-sky projection $I(x, y)$ of any intrinsic quantity $G(\xi)$, can be represented as:

$$
\begin{aligned}
I(x, y) & =\int_{0}^{\infty} G(\xi) \mathrm{d} z \\
& =\int_{\eta}^{\infty} G(\xi) \frac{\mathrm{d} \xi}{\sqrt{\xi-\eta^{2}}}
\end{aligned}
$$

where

$$
\eta=x^{2}+y^{2} \frac{q_{1}^{2} q_{2}^{2}}{R^{2}} .
$$

The notation here is chosen to concur with that in Fabricant et al. (1984). Since the RHS of this equation is a function of $\eta$ only and the projected quantity $I(x, y)$ is a constant along the isophotes, the equation relating $\eta$ to $x$ and $y$ must give the form of the isophotes. Then the ratio $q_{\mathrm{p}}$, of the extent along the $x$-axis to that along the $y$-axis is related to ratio of the semi-axes along $X, Y$ and $Z$-axes respectively as:

$q_{\mathrm{p}}^{2}=\frac{q_{1}^{2} q_{2}^{2}}{q_{2}^{2} \cos ^{2}(i)+q_{1}^{2} \sin ^{2}(i)}$.

A similar exercise involving integration over $y$ yields the ratio $e_{\mathrm{LOS}}$ between the extent along the $z$-axis and the $x$-axis as:

$e_{\mathrm{LOS}}^{2}=\frac{\frac{1}{q_{1}^{2}} \frac{1}{q_{2}^{2}}}{\frac{\cos ^{2}(i)}{q_{1}^{2}}+\frac{\sin ^{2}(i)}{q_{2}^{2}}}$.

The cluster elongation index $q_{\mathrm{LOS}}$ that is used elsewhere in the paper (notation borrowed from De Filippis et al. 2005) differs from $e_{\mathrm{LOS}}$ in that $q_{\mathrm{LOS}}$ is the ratio along the LOS to the photometric major axis.

Thus, for oblate systems, when $q_{1}=1, q_{2}=q>1, q_{\mathrm{p}}>1$ and $q_{\mathrm{LOS}}=e_{\mathrm{LOS}}$,

$q_{\mathrm{p}}^{2}=\frac{q^{2}}{q^{2} \cos ^{2}(i)+\sin ^{2}(i)}$.

Using this in Eq. (A.6), we get that for oblate systems,

$q_{\mathrm{LOS}}^{2}=\frac{1-q_{\mathrm{p}}^{2} \cos ^{2}(i)}{\sin ^{2}(i)}$.

For prolate systems, $q_{1}=1, q_{2}<1 \Longrightarrow q_{2}=1 / q, q_{\mathrm{p}}<1$ and $q_{\mathrm{LOS}}=e_{\mathrm{LOS}} / e_{\mathrm{p}}$. So,

$q_{\mathrm{p}}^{2}=\cos ^{2}(i)+q^{2} \sin ^{2}(i)$.

Using this in Eq. (A.6), we get that for prolate systems,

$q_{\mathrm{LOS}}^{2}=\frac{q_{\mathrm{p}}^{2}-\cos ^{2}(i)}{q_{\mathrm{p}}^{4} \sin ^{2}(i)}$.
Since $q_{\mathrm{p}}$ and $q_{\mathrm{LOS}}$ are observables, $q_{1}$ and $q_{2}$ can be calculated for the three different types of triaxial systems, as long as we keep in mind that:

- for Triaxial-type I: $q_{1}<1, q_{2}>1, q_{\mathrm{p}}<1$ and $q_{\mathrm{LOS}}=$ $e_{\mathrm{LOS}} / q_{\mathrm{p}}$;

- for Triaxial-type II: $q_{1}<1, q_{2}>1, q_{\mathrm{p}}>1$ and $q_{\mathrm{LOS}}=e_{\mathrm{LOS}}$;

- for Triaxial-type III: $q_{1}>1, q_{2}<1, q_{\mathrm{p}}>1$ and $q_{\mathrm{LOS}}=e_{\mathrm{LOS}}$.

We use Eqs. (A.5) and (A.6) to determine $q_{1}$ and $q_{2}$ in a triaxial configuration. To do this, the values of $q_{\mathrm{p}}$ and $q_{\mathrm{LOS}}$ will of course need to be supplied from observations. The value of $q_{\mathrm{LOS}}$ that we consider is the centroid of the reported error band in De Filippis et al. (2005).

However, for the different types of triaxial systems that we consider, there are two other constraints involving $q_{1}$ and $q_{2}$ (in the form of inequalities); such inequalities are typical of the triaxial type of the cluster at hand and are listed above for types I, II and III. Implementing these would imply two distinct ranges of inclinations for each cluster. The overlap of these provides the final constraints on the inclinations. Once this final range of inclinations is obtained, Eqs. (A.5) and (A.6) are solved to give $q_{1}$ and $q_{2}$, at the median of the recovered inclination range.

\section{References}

Bailin, J., \& Steinmetz, M. 2005, ApJ, 627, 647 Bendinelli, O. 1991, ApJ, 366, 599

Bissantz, N., \& Munk, A. 2001, A\&A, 376, 735

Chakrabarty, D., \& Saha, P. 2001, AJ, 122, 232

Chakrabarty, D., \& Ferrarese, L. 2008, Int. J. Mod. Phys. D, as part of proceedings for the 6th International Workshop on Data Analysis in Astronomy, Modelling and Simulations in Science, 17, 2

Cole, S., \& Lacey, C. 1996, MNRAS, 281, 716

De Filippis, E., Sereno, M., \& Bautz, M. W. 2005, Adv. Space Res., 36, 715 De Filippis, E., Sereno, M., Bautz, M. W., \& Longo, G. 2005, ApJ, 625, 108 Dubinski, J., \& Carlberg, R. G. 1991, ApJ, 378, 496

Ebeling, H., Voges, W., Bohringer, H., et al. 1996, MNRAS, 281, 799

Fabian, A. C., Hu, E. M., Cowie, L. L., \& Grindlay, J. 1981, ApJ, 248, 47

Fabricant, D., Rybicki, G., \& Gorenstein, P. 1984, ApJ, 286, 186

Frenk, C. S., White, S. D. M., Davis, M., \& Efstathiou, G. 1988, ApJ, 327, 507 Gottlöber, S., \& Yepes, G. 2007, ApJ, 664, 117

Hayashi, E., Navarro, J. F., \& Springel, V. 2007, MNRAS, 377, 50

Hughes, J. P., \& Birkinshaw, M. 1998, ApJ, 501, 1

Jing, Y. P., \& Suto, Y. 2002, ApJ, 574, 538

Kasun, S. F., \& Evrard, A. E. 2005, ApJ, 629, 781

Mahdavi, A., Hoekstra, H., Babul, A., et al. 2007, ApJ, 664, 162

Mason, B. S., \& Myers, S. T. 2000, ApJ, 540, 614

Mason, B. S., Myers, S. T., \& Readhead, A. C. S. 2001, ApJ, 555, L11

Palmer, P. L. 1994, MNRAS, 266, 697

Pizzolato, F., Molendi, S., Ghizzardi, S., \& De Grandi, S. 2003, ApJ, 592, 62

Plionis, M., Basilakos, S., \& Tovmassian, H. M. 2004, MNRAS, 352, 1323

Reblinsky, K. 2000, A\&A, 364, 377

Reese, E. D., Carlstrom, J. E., Joy, M., et al. 2002, ApJ, 581, 53

Sand, D. J., Treu, T., \& Ellis, R. S. 2002, ApJ, 574, L129

Sereno, M., De Filippis, E., Longo, G., \& Bautz, M. W. 2006, ApJ, 645, 170

Voit, G. M., Bryan, G. L., Balogh, M. L., \& Bower, R. G. 2002, ApJ, 576, 601

Warren, M. S., Quinn, P. J., Salmon, J. K., \& Zurek, W. H. 1992, ApJ, 399, 405

Yoshikawa, K., \& Suto, Y. 1999, ApJ, 513, 549

Zaroubi, S., Squires, G., Hoffman, Y., \& Silk, J. 1998, ApJ, 500, L87

Zaroubi, S., Squires, G., de Gasperis, G., et al. 2001, ApJ, 561, 600 